\title{
A Covariant Generalization of the Real-Time Green's Functions Method in the Theory of Kinetic Equations
}

\author{
S. A. Smolyansky and A. V. Prozorkevich \\ Physics Department, Saratov State University, 410071, Saratov, Russia \\ G. Maino \\ ENEA, Applied Physics Division, via Don G. Fiammelli 2, 40129, Bologna, Italy \\ and \\ S. G. Mashnik \\ T-2, Theoretical Division, Los Alamos National Laboratory, Los Alamos, NM 87545
}

\begin{abstract}
A generalized quantum kinetic equation (RKE) of the Kadanoff-Baym type is obtained on the basis of the Heisenberg equations of motion where the time evolution and space translation are separated from each other by means of the covariant method. The same approach is used also for a covariant modification of the real-time Green's functions method based on the Wigner representation. The suggested approach does not contain arbitrariness' elements and uncertainties which often arise from derivation of RKE on the basis of the motion equations of the Kadanoff-Baym type for the correlation functions in the case of systems with inner degrees of freedom.

Possibilities of the proposed method are demonstrated by examples of derivation of RKE of the Vlasov type and collision integrals of the Boltzmann-Uehling-Uhlenbeck (BUU) type in the frame of the $\sigma \omega$-version of quantum hadrodynamics, for the simplest case of spin saturated nuclear matter without antinuclear component. Here, the quasiparticle approximation in a covariant performance is used. A generalization of the method for the description of strong non-equilibrium states based on the non-equilibrium statistical operator is then proposed as well.
\end{abstract}

\section{Introduction}

The recent growing interest in the relativistic nuclear physics, especially at intermediate energies, has stimulated the development of the theory of relativistic kinetic equations (RKE) as well. In particular, the main problems of dynamical RKE derivation were discussed within the framework of different approaches with well grounded traditions in the non-relativistic case, namely the method of the real-time (contour) Green's functions [1]-[6], the BBGKY method (method of many particles correlation functions) [7], 8], the method of the non-equilibrium statistical operator [9, 10], and several other techniques [11-15].

However, in some approaches, the transition to the relativistic region it is not obvious and can lead to misleading results. This situation was analyzed in Refs. [16]-17] on a rather simple quantum field model which describes interaction of the fermion and boson 
subsystems in the mean field approximation. In these works, it was shown that the equations of motion for a two-point correlation function lead to true RKE of the Vlasov type only if one uses some definite simple rules of projection. It is important that the same RKE can be derived also by a direct method based on the Heisenberg equation of motion [9, 10], 16]-22]. In the latter approach, any ambiguities in the RKE derivation are thus in general avoided. These features of the direct method represent a suitable expedient to obtain a correct generalized RKE in the frame of a relativistic analogue of the Kadanoff-Baym formalism. This is the main result of our study.

Above-mentioned works [9, 10], [16]-22] are devoted to covariant generalizations of the Zubarev method of non-equilibrium statistical operator [47] in terms of the relativistic Wigner functions. However, this formalism presents some technical difficulties to go beyond the Born approximation, while the formalism introduced in this work, based on the real-time Green function, avoids this kind of mathematical troubles.

In order to solve this main problem, we develop a specific relativistic modification of the real-time Green's functions method in the Wigner representation. This improvement proves to be possible since the transition to the Wigner representation in relativistic theory allows us to introduce a time-like direction which can be connected with the momentum argument of the Wigner function. As a result, the time variable determining the evolution of the system can be defined as a true scalar, which plays the role of the proper time in a given point of the eight-dimensional phase space. This invariant time is used also to define the chronological ordering operation in the real-time Green's functions . It leads to an obvious modification of this method at all stages of the formalism including the description of the dispersion properties of quantum field systems. Such a modification of the real-time Green's functions method is convenient also in order to describe the dispersion properties of a quantum-field system at finite temperature and density as it proves to be obviously relativistic invariant for all the steps of the relevant calculations.

The outline of the paper is as follows. In Sec. 2, we discuss shortly the "direct way" of the derivation of RKE in terms of one-particle relativistic Wigner's functions. The advantages of this approach are its simplicity and clearness. This fact originates from the use of the Heisenberg picture from the very beginning. However, in this section, we do not discuss any truncation scheme and derivation of closed-form RKE. This choice is convenient for a comparison with the method based on the Kadanoff-Baym motion equations for correlation functions (Sec. 3). A covariant modification of the real-time Green's functions method is found as an adequate formalism for this aim. As a result, we obtain a generalized RKE of the Kadanoff-Baym type without using any irrelevant assumption, thus allowing us to use effectively the suggested method for a reliable kinetic description of a quite wide class of systems with inner degrees of freedom.

In essence, the only limitation of the suggested method is a request of a polynomial character of interaction of systems under study. It is important that the suggested approach results in a self-consistent, non-contradictory, and unambiguous theory of kinetic equations. To illustrate the possibilities of the theory, two examples are discussed in detail. Firstly, a RKE of the Vlasov type (the mean field approximation, Sec. 2) and then a collision integral of the BUU type (the quasiparticle approximation, Sec. 4). The standard Walecka model, which is a quantum field model of the relativistic nuclear matter consisting of nucleons and two types (scalar and vector) of mesons (quantum hadrodynamics) is used in our work as a toy model.

In Sec. 5, we consider another generalization of relativistic kinetic theory based on a change of the standard averaging procedure under the equilibrium density matrix by an averaging using the non-equilibrium statistical operator. This step leads to a possible kinetic description of strongly non-equilibrium states which are often met in the relativistic 
nuclear physics. Finally, conclusions are drawn in Sec. 6. Everywhere, we work with natural units, $\hbar=c=1 \mathrm{c}$.

\section{The motion equation for the Wigner function}

To illustrate our approach on a nontrivial example of a system with internal degrees of freedom, let us consider the kinetic description of the Fermi subsystem of a quantum-field system. For a consistent dynamical construction of the relativistic kinetic theory, we will start with the introduction of the one-particle Wigner function of the Fermi subsystem,

$$
f_{\alpha \beta}(x, p)=(2 \pi)^{-4} \int d y e^{-i p y}<P_{\alpha \beta}(x, y)>,
$$

where the symbol $<\ldots>=\operatorname{Tr} . . \rho$ denotes the operation of statistical averaging with density matrix $\rho$ in the Heisenberg representation and

$$
P_{\alpha \beta}(x, y)=\bar{\psi}_{\beta}\left(x_{+}\right) \psi_{\alpha}\left(x_{-}\right),
$$

$\psi(x)$ and $\bar{\psi}(x)$ being the usual field operators, and $x_{ \pm}=x \pm y / 2$.

In the realm of dynamical basis of the theory, we choose the Heisenberg equations of motion, namely,

$$
i \partial^{\mu} A(x)=\left[A(x), P^{\mu}\right]
$$

where $A(x)=A[x, \psi(x), \bar{\psi}(x)]$ is an arbitrary local operator and $P^{\mu}$ is the total 4momentum of the system,

$$
P^{\mu}=\int d \sigma_{\nu}(x \mid n) T^{\mu \nu}
$$

Here, $d \sigma_{\nu}(x \mid n)$ is a vector element of an arbitrary space-like hyperplane, $\sigma(n)$, with a time-like normal vector, $n^{\mu}\left(n^{2}=1\right)$, and $T^{\mu \nu}=T^{\nu \mu}$ is the energy-momentum tensor..

The energy and momentum conservation laws lead to the independence of the integrals (四) from the selection of a given hyperplane $\sigma(n)$, and we can fix the time-like direction by means of an external condition (a relevant example will be given below).

In order to describe the dynamics of the system along the time-like direction, $n^{\mu}$, and the space translation along the independent space-like directions on the hyperplane, $\sigma(n)$, a boost transformation with the "velocity", $n^{\mu}$, can be used for the motion Eqs. (3). Technically, this result is achieved using the projection of Eqs. (3) on the direction $n^{\mu}$ and on the hyperplane, $\sigma(n)$. Convolution of Eqs. (3) with the vector, $n^{\mu}$, leads to the following dynamical equation [23],

$$
i \frac{\partial A(x)}{\partial \tau}=[A(x), H(\tau)] .
$$

The parameter,

$$
\tau=n_{\mu} x^{\mu}
$$

plays here the role of a proper time in a new coordinate system. In Eq.(5), the derivative along the direction of $n^{\mu}$ is thus introduced,

$$
\frac{\partial}{\partial \tau}=n^{\mu} \frac{\partial}{\partial x^{\mu}}
$$

$H(\tau)$ is the scalar Hamiltonian of the system and reads as

$$
H(\tau)=n_{\mu} P^{\mu}=\int d \sigma(n) n_{\mu} T^{\mu \nu} n_{\nu}
$$


because $d \sigma^{\mu}(x \mid n)=n^{\mu} d \sigma(x \mid n)$. This procedure of a formal restoration of the relativistic invariance in the description of the time evolution is very popular in the quantum field theory (see, e.g., 23]).

The projection of Eqs. (3) on the space-like directions is carried out by the convolution of Eqs. (3) with the projection operator,

$$
\Delta^{\mu \nu}=g^{\mu \nu}-n^{\mu} n^{\nu}, \quad n_{\mu} \Delta^{\mu \nu}=0 .
$$

The resulting equations provide a description of infinitesimal transforms of the system on the space-like hyperplane, $\sigma(n)$. These equations are not interesting for the present aim of developing a kinetic theory [16]-17].

Now, we can write the motion equation for the Wigner function (11). Performing the $\tau$-differentiation of function (1) and using the Liouville equation in the Heisenberg representation,

$$
\frac{d \rho}{d \tau}=0
$$

after substitution of the motion equation (5) and using the definition (7), we find:

$$
\frac{\partial f(x, p)}{\partial \tau}=n^{\mu} \frac{\partial}{\partial x^{\mu}} f(x, p)=-i(2 \pi)^{-4} \int d y e^{-i p y}<[P(x, y), H(\tau)]>.
$$

It is worth remarking that, for sake of convenience, the spin indices are dropped out in our notation.

At this stage, we can remove the arbitrariness in the choice of the unit time-like vector, $n^{\mu}$, using the specific character of the mixed Wigner's representation. Let us assume that the momentum vector, $p^{\mu}$, in Eq. (11) is a time-like one (Assumption 1). Then, we can define a unit vector in the direction of $p^{\mu}$ and identify it with the vector, $n^{\mu}$ (Assumption 2) [9, 10], [16]-17]:

$$
n^{\mu} \stackrel{\text { def }}{=} u^{\mu}=p^{\mu} / \sqrt{p^{2}}, \quad u^{2}=1 .
$$

Assuming a description of non-equilibrium quantum field systems in terms of the Wigner functions (1), often one limits oneself to consider only processes on the mass shell,

$$
p^{2}=m^{2}
$$

In this respect, introduction of Assumptiones 1 and 2 does not produce any essential restriction even at the off mass shell. In other words, Assumption 1 extracts the physical significant domain, $\mathcal{P}^{+}$, from the full phase space, $\mathcal{P} \equiv(x, p)$. Let us remark that an analogous restriction is usually introduced at the stage of the definition of Wigner's functions of the states with positive and negative energies [26]. On the other hand, Assumption 1 can be taken off and go out into the space-like region (see the discussion at the end of Sec. 3). We conserve this restriction here in order to remain into the framework of the ordinary dynamical description.

The effectiveness of the introduction of Assumptiones 1 and 2 is confirmed by the fact that substitution of the relation (12) into Eq. (11) completely removes any arbitrariness in this equation and leads to a "generalized RKE",

$$
\begin{gathered}
p_{\mu} \partial^{\mu}(x) f(x, p)=-i(2 \pi)^{-4} \sqrt{p^{2}} \int d y e^{-i p y}<[P(x, y), H(\tau)]>= \\
=-i(2 \pi)^{-4} \sqrt{p^{2}} \int d y e^{-i p y}<\left[\bar{\psi}\left(x_{+}\right), H\left(\tau_{+}\right)\right] \psi\left(x_{-}\right)+\bar{\psi}\left(x_{+}\right)\left[\psi\left(x_{-}\right), H\left(\tau_{-}\right)\right]>
\end{gathered}
$$

where we took into account the arbitrariness of the assigned hyperplane into the Hamiltonian (8) $H(\tau)=H\left(\tau_{+}\right)=H\left(\tau_{-}\right)$. Essentially, this is the first equation of the BBGKY 
hierarchy in the Wigner representation. In the mean field approximation, Eq. (14) results to be a non-contradictory RKE of the Vlasov type [16]-[17], [21, 22].

In a general case, it is necessary to introduce different truncation schemes to obtain closed-form RKE from Eq. (14). The usual perturbation theory on the coupling constant starting from Eq. (14) was developed in Refs. [9, 10], [18]-[20]. Within the framework of such a perturbation theory, two types of RKE with collision integrals of the second order have been obtained: RKE of the Bloch type (for the vertices of the "three-tails" type) and RKE of the Boltzmann type (for the effective vertices of the "four-tails" type). The Green's functions method offers an effective way to go beyond the perturbation approach. In the following Section, Eq.(14) will be re-formulated in terms of modified contour Green's functions. In particular, this will allow us to get a unambiguous generalized RKE of the Kadanoff-Baym type, thus opening some perspectives to go beyond the standard perturbation theory without breakdown of the relativistic invariance of kinetic theory.

\section{Generalized RKE in terms of modified real-time Green's functions}

\subsection{Covariant real-time Green's functions}

The modern technique of derivation of the Kadanoff-Baym type kinetic equations is based on the real-time Green's functions method [1, 2, 3, 6, 24, 25]. Now, our aim is to combine together the " generalized RKE"(14) and a system of motion equations of the KadanoffBaym type for the real-time Green's functions. As a first step, we will modify this method taking into account the existence of a preferred time-like direction (Assumptiones 1 and 2 in Sec. 2) in the definition of the Green's functions in the Wigner representation. This direction is fixed by relation (12) and leads to scalar time variables (see Eq. (6)),

$$
\tau_{i}=x_{i}^{\mu} n_{\mu}, \quad n^{\mu} \rightarrow u^{\mu}
$$

where $x_{i}^{\mu}$ are arguments of the two-points Green's functions. It is implied that identification (12) is fulfilled after a transition to the Wigner representation. The time arguments (15) are convenient for use in the definition of the ordering operation determining the modified real-time Green's functions. Limiting ourselves to the case of a Fermi subsystem, let us introduce the following Green's functions in the Wigner representation,

$$
G_{\alpha \beta}^{(k)}(x p)=(2 \pi)^{-4} \int d y e^{i p y} G_{\alpha \beta}^{(k)}\left(x_{+}, x_{-}\right),
$$

where index $(\mathrm{k})$ enumerates the elements of the real-time (contour) Green's function,

$$
\begin{aligned}
& G_{\alpha \beta}^{c}\left(x_{1}, x_{2}\right)=-i<T^{c}\left[\psi_{\alpha}\left(x_{1}\right) \bar{\psi}_{\beta}\left(x_{2}\right)\right]>=G_{12}^{--}, \\
& G_{\alpha \beta}^{a}\left(x_{1}, x_{2}\right)=-i<T^{a}\left[\psi_{\alpha}\left(x_{1}\right) \bar{\psi}_{\beta}\left(x_{2}\right)\right]>=G_{12}^{++}, \\
& G_{\alpha \beta}^{>}\left(x_{1}, x_{2}\right)=-i<\psi_{\alpha}\left(x_{1}\right) \bar{\psi}_{\beta}\left(x_{2}\right)>=G_{12}^{+-}, \\
& G_{\alpha \beta}^{<}\left(x_{1}, x_{2}\right)=i<\bar{\psi}_{\beta}\left(x_{2}\right) \psi_{\alpha}\left(x_{1}\right)>=G_{12}^{-+},
\end{aligned}
$$

and $\left(T^{a}\right) T^{c}$ represents (anti)chronological time ordering along the $\tau$-axis:

$$
\begin{array}{ll}
T^{c}\left[A\left(x_{1}\right) B\left(x_{2}\right)\right] & \stackrel{\text { def }}{=} \theta\left(\tau_{1}-\tau_{2}\right) A\left(x_{1}\right) B\left(x_{2}\right)-\theta\left(\tau_{2}-\tau_{1}\right) B\left(x_{2}\right) A\left(x_{1}\right), \\
T^{a}\left[A\left(x_{1}\right) B\left(x_{2}\right)\right] \stackrel{\text { def }}{=} \theta\left(\tau_{2}-\tau_{1}\right) A\left(x_{1}\right) B\left(x_{2}\right)-\theta\left(\tau_{1}-\tau_{2}\right) B\left(x_{2}\right) A\left(x_{1}\right) .
\end{array}
$$


The operators, $A(x)$ and $B(x)$, are selected from the set of the field operators, $\bar{\psi}(x)$ and $\psi(x)$.

The path-ordered real-time contour Green's functions are defined by the relation

$$
G\left(x_{1}, x_{2}\right)=-i<T_{c}\left[\psi\left(x_{1}\right) \bar{\psi}\left(x_{2}\right)\right]>,
$$

where $T_{c}$ is the path-ordering operator on the $\tau$-contour which is chosen in the usual way (see Fig. 1).

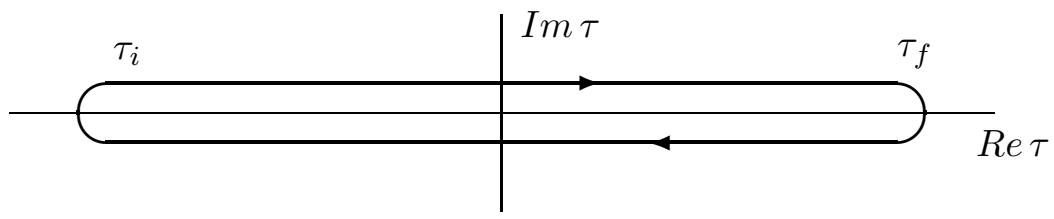

Fig. 1. The Keldysh-Schwinger contour, $C$, with the upper $C^{+}$and lower $C^{-}$branches.

It is worth stressing that this definition is based on the Wigner representation (16) and Assumptiones 1 and 2. This restriction leads by itself to a break-down of a simple analogy between the Wigner and the Fourier transforms.

Let us return to Eq. (14). From the definitions, (11) and (16,17), it follows that

$$
G^{<}(x p)=i f(x p) \text {. }
$$

Therefore, Eq. (14) can be rewritten in the form

$$
p^{\mu} \partial_{\mu}(x) G^{<}(x p)=(2 \pi)^{-4} \sqrt{p^{2}} \int d y e^{-i p y}<[P(x, y), H(\tau)]>.
$$

Let us assume now that the Hamiltonian of the fermion subsystem has a polynomial structure and adopt the usual notation,

$$
H(\tau)=H_{0}(\tau)+H_{i n}(\tau), \quad H_{i n}(\tau)=H_{M F}(\tau)+H_{r}(\tau) .
$$

Here, $H_{0}(\tau)$ describes the free evolution of the fermion field,

$$
H_{0}(\tau)=-\int d \sigma(x \mid u) \bar{\psi}(x)\left\{\frac{i}{2} \gamma^{\mu} \stackrel{\leftrightarrow}{\partial_{\mu}^{\perp}}(x)-m\right\} \psi(x)
$$

where $\partial_{\mu}^{\perp}(x)$ is the space-like derivative,

$$
\partial_{\mu}^{\perp}(x)=\Delta_{\mu}^{\nu} \partial_{\nu}(x),
$$

and $\Delta^{\mu \nu}$ is the projection operator (9). In the interaction Hamiltonian, $H_{i n}(\tau)$, Eq. (22), the $H_{M F}(\tau)$ part, corresponding to the mean field approximation, is explicitly separated. Then, $H_{r}(\tau)$ describes the residual part of the interaction.

It is easy to calculate the free motion part in the right-hand side of Eq. (21) for the Hamiltonian (23). For covariant transformations, it is convenient to use the following rule,

$$
\int d \sigma\left(x^{\prime} \mid u\right) S\left(x-x^{\prime}\right) y\left(x^{\prime}\right)=i u_{\mu} \gamma^{\mu} y(x), \quad x \in \sigma(u),
$$

where $y(x)$ is an arbitrary function of field operators and $S(x)$ is the anti-commutation function of the fermion fields, namely $S\left(x-x^{\prime}\right)=i\left[\psi(x), \bar{\psi}\left(x^{\prime}\right)\right]_{+}$. Relation (25) is based 
on a covariant generalization of the well-known property of the function $S(x)$ (see, e.g., [26])

$$
\left.S(x)\right|_{x^{0}=0}=i \gamma^{0} \delta^{(3)}(x),
$$

(a simple proof of Eq. (25) is given in Appendix A). This kind of calculation for Eq. (21) leads to the following result:

$$
\begin{aligned}
& p^{\mu} \partial_{\mu}(x) G^{<}(x p)+\frac{1}{2}\left[\hat{p} \gamma^{\mu}, \partial_{\mu}(x) G^{<}(x p)\right]+i m\left[\hat{p}, G^{<}(x p)\right]= \\
& =(2 \pi)^{-4} \sqrt{p^{2}} \int d y e^{-i p y}<\left[\bar{\psi}\left(x_{+}\right), H_{i n}\left(\tau_{+}\right)\right] \psi\left(x_{-}\right)+\bar{\psi}\left(x_{+}\right)\left[\psi\left(x_{-}\right), H_{i n}\left(\tau_{-}\right)\right]>,
\end{aligned}
$$

where $\hat{p}=p^{\mu} \gamma_{\mu}$.

The following step consists in the search of a connection between the right-hand side of Eq. (27) and the mass operators, $\Sigma^{(k)}\left(x_{1}, x_{2}\right)$, defined on the $\tau$-contour. To this aim, let us write the motion equations for the real-time Green's functions,

$$
\begin{aligned}
{\left[i \gamma \partial\left(x_{1}\right)-m\right] G\left(x_{1}, x_{2}\right) } & =\delta_{c}\left(x_{1}, x_{2}\right)+\int_{c} d^{4} x^{\prime} \Sigma\left(x_{1}, x^{\prime}\right) G\left(x^{\prime}, x_{2}\right) \\
G\left(x_{1}, x_{2}\right)\left[-i \gamma \overleftarrow{\partial}\left(x_{2}\right)-m\right] & =\delta_{c}\left(x_{1}, x_{2}\right)+\int_{c} d^{4} x^{\prime} G\left(x_{1}, x^{\prime}\right) \Sigma\left(x^{\prime}, x_{2}\right)
\end{aligned}
$$

The integration in the right-hand side of Eqs. (28) is performed according to the rules:

$$
\int_{c} d^{4} x \ldots=\int d \sigma(x \mid u) \int_{c} d \tau \ldots
$$

and

$$
\int_{c} d \tau \ldots=\left.\int_{t_{0}}^{\infty} d \tau \ldots\right|_{c^{+}}-\left.\int_{t_{0}}^{\infty} d \tau \ldots\right|_{c^{-}}
$$

where $c^{+}\left(c^{-}\right)$is the upper (lower) branch of the $\tau$-contour (see Fig. 1). Finally, the delta function on the $\tau$-contour is defined as

$$
\delta_{c}\left(x_{1}, x_{2}\right)=\delta_{\sigma}\left(x_{1}, x_{2}\right) \delta_{c}\left(\tau_{1}-\tau_{2}\right),
$$

where

$$
\delta_{c}\left(\tau_{1}-\tau_{2}\right)=\left\{\begin{array}{rcc}
\delta\left(\tau_{1}-\tau_{2}\right) & \text { if } & \tau_{1} \in c^{+} \text {and } \tau_{2} \in c^{+} \\
-\delta\left(\tau_{1}-\tau_{2}\right) & \text { if } & \tau_{1} \in c^{-} \text {and } \tau_{2} \in c^{-} \\
0 & & \text { otherwise }
\end{array}\right.
$$

$\delta_{\sigma}(x)$ is the $d=3$ delta function of the space arguments on the hyperplane $\sigma(x \mid u)$.

For purpose of comparison with Eq. (27), let us write the motion equations for the correlation function, $G^{<}\left(x_{1}, x_{2}\right)$. From Eqs. (28), the Kadanoff-Baym type equations follow

$$
\begin{aligned}
& {\left[i \gamma \partial\left(x_{1}\right)-m-\Sigma_{M F}\left(x_{1}\right)\right] G^{<}\left(x_{1}, x_{2}\right)=\int d x^{\prime}\left\{\Sigma^{<}\left(x_{1}, x^{\prime}\right) G^{A}\left(x^{\prime}, x_{2}\right)+\Sigma^{R}\left(x_{1}, x^{\prime}\right) G^{<}\left(x^{\prime}, x_{2}\right)\right\}} \\
& G^{<}\left(x_{1}, x_{2}\right)\left[-i \gamma \overleftarrow{\partial}\left(x_{2}\right)-m-\Sigma_{M F}\left(x_{2}\right)\right]=\int d x^{\prime}\left\{G^{<}\left(x_{1}, x^{\prime}\right) \Sigma^{A}\left(x^{\prime}, x_{2}\right)+G^{R}\left(x_{1}, x^{\prime}\right) \Sigma^{<}\left(x^{\prime}, x_{2}\right)\right\}
\end{aligned}
$$

Here, the mean field part of the mass operator was extracted according to the following prescription:

$$
\begin{aligned}
\Sigma\left(x_{1}, x_{2}\right) & =\Sigma_{M F}\left(x_{1}\right) \delta\left(x_{1}-x_{2}\right)+ \\
& +\theta\left(\tau_{1}-\tau_{2}\right) \Sigma^{>}\left(x_{1}, x_{2}\right)+\theta\left(\tau_{2}-\tau_{1}\right) \Sigma^{<}\left(x_{1}, x_{2}\right) .
\end{aligned}
$$


Moreover, the retarded and advanced Green's functions were introduced,

$$
\begin{aligned}
G^{R / A}\left(x_{1}, x_{2}\right) & = \pm \theta\left[ \pm\left(\tau_{1}-\tau_{2}\right)\right]\left\{G^{>}\left(x_{1}, x_{2}\right)-G^{<}\left(x_{1}, x_{2}\right)\right\}= \\
& =G^{c}\left(x_{1}, x_{2}\right)-G^{</>}\left(x_{1}, x_{2}\right)=G^{>/<}\left(x_{1}, x_{2}\right)-G^{a}\left(x_{1}, x_{2}\right)
\end{aligned}
$$

In a similar way, the retarded and advanced components of the mass operator are defined. A mutual exchange of the symbols $>$ and $<$ transforms Eqs. (33) and (34) into the corresponding equations for the correlations functions, $G^{>}\left(x_{1}, x_{2}\right)$. By this way, the Kadanoff-Baym equations (33) and (34) have the usual form however they are defined using covariant modified constituents (Green's functions and mass operators). However, these small corrections are essential for the search of the agreement with RKE (27).

The transition to the mixed Wigner's representation for the motion Eqs. (33) and (34) is usually done with a limitation to the lowest orders of the gradient decomposition (the first order into the drift part of RKE and order zero for the collision part) 229], approximation that is justified only for rather slow kinetic processes. This allows us to write the motion equations in the simplest local form. This restriction casts some doubt upon the description of non-equilibrium processes in nuclear matter at extreme conditions (see, e.g., [27, 28]). However, for simplicity's sake, we keep here this approximation only.

Then, the motion Eqs. (33) and (34) in the Wigner representation are transformed into the following equations:

$$
\begin{array}{r}
{\left[\hat{p}+\frac{i}{2} \gamma \partial(x)-m-\Sigma_{M F}(x)+\frac{i}{2} \partial_{\mu}(x) \Sigma_{M F}(x) \partial^{\mu}(p)\right] G^{<}(x p)=} \\
=\Sigma^{<}(x) G^{A}(x p)+\Sigma^{R}(x) G^{<}(x p), \\
G^{<}(x p)\left[\hat{p}-\frac{i}{2} \gamma \overleftarrow{\partial}(x)-m-\Sigma_{M F}(x)-\frac{i}{2} \overleftarrow{\partial}_{\mu}(p) \partial^{\mu}(x) \Sigma_{M F}(x)\right]= \\
=G^{<}(x p) \Sigma^{A}(x p)+G^{R}(x p) \Sigma^{<}(x p)
\end{array}
$$

We have neglected gradient terms in the right-hand sides of these equations 29].

\subsection{Generalized RKE}

Let us fulfill now the concordance of the "generalized RKE" (14) and the system of equations of the Kadanoff-Baym type (37) and (38). Obviously, for this aim, it is necessary to find such a combination of these equations which provides a coincidence with the left-hand side of Eq. (27) describing the free evolution of the fermion subsystem. This assumption will permit us to identify (in the corresponding orders of the gradient expansion) also the other parts of Eq. (27) and Eqs. (37), (38).

In order to perform such a construction, it is sufficient to multiply Eq. (38) by $i \hat{p}$ from the right and Eq. (37) from the left and to subtract the second result from the first one, i.e., it is necessary to consider the following algebraic combination:

$$
E q .(38) i \hat{p}-i \hat{p} E q \cdot(37) \text {. }
$$

Then, we get the following equation

$$
\begin{array}{r}
p^{\mu} \partial_{\mu}(x) G^{<}(x p)+\frac{1}{2}\left[\hat{p} \gamma_{\mu}, \partial^{\mu}(x) G^{<}(x p)\right]+i m\left[\hat{p}, G^{<}(x p)\right]- \\
-i\left\{G^{<}(x p) \Sigma_{M F}(x) \hat{p}-\hat{p} \Sigma_{M F}(x) G^{<}(x p)\right\}+ \\
+\frac{1}{2}\left\{\partial_{\mu}(p) G^{<}(x p) \partial^{\mu}(x) \Sigma_{M F}(x) \hat{p}+\hat{p} \partial_{\mu}(x) \Sigma_{M F}(x) \partial^{\mu}(p) G^{<}(x p)\right\}=-S(x p),
\end{array}
$$


where $S(x p)$ is the collision integral,

$$
\begin{aligned}
S(x p)= & i \hat{p}\left\{\Sigma^{<}(x p) G^{A}(x p)+\Sigma^{R}(x p) G^{<}(x p)\right\}- \\
& -i\left\{G^{<}(x p) \Sigma^{A}(x p)+G^{R}(x p) \Sigma^{<}(x p)\right\} \hat{p} .
\end{aligned}
$$

Now, from a comparison of Eqs. (27) and (40), it follows an equivalence of the left and right parts of those relations:

$$
\begin{gathered}
(2 \pi)^{-4} \sqrt{p^{2}} \int d y e^{-i p y}<\left[\bar{\psi}\left(x_{+}\right), H_{M F}\left(\tau_{+}\right)\right] \psi\left(x_{-}\right)+\bar{\psi}\left(x_{+}\right)\left[\psi\left(x_{-}\right), H_{M F}\left(\tau_{-}\right)\right]>= \\
=i\left\{G^{<}(x p) \Sigma_{M F}(x) \hat{p}-\hat{p} \Sigma_{M F}(x) G^{<}(x p)\right\}- \\
-\frac{1}{2}\left\{\partial_{\mu}(p) G^{<}(x p) \partial^{\mu}(x) \Sigma_{M F}(x) \hat{p}+\hat{p} \partial_{\mu}(x) \Sigma_{M F}(x) \partial^{\mu}(p) G^{<}(x p)\right\}
\end{gathered}
$$

Let us remind that these equalities are correct only in the lowest orders of the gradient expansions.

Both parts of the equality (42) describe the force contribution (with the inverse signs) of the mean fields into the convective part of RKE (27) or (40). Let us illustrate this aspect by means of an actual model. We thus refer to the standard Walecka model, where the Hamiltonian for the interaction of nucleons with massive vector and scalar meson fields reads as [30]:

$$
H_{i n}(\tau)=\int d \sigma(x \mid u) \bar{\psi}(x)\left\{g_{v} \omega^{\mu}(x) \gamma_{\mu}-g_{s} \phi(x)\right\} \psi(x) .
$$

In the mean field approximation, the relevant Hamiltonian, $H_{M F}(\tau)$, can be deduced from this one by replacing the meson fields operators with their mean values, $\phi \rightarrow\langle\phi\rangle$, and $\omega^{\mu} \rightarrow<\omega^{\mu}>$. Then, the corresponding contribution to the mass operator is equal to

$$
\Sigma_{M F}(x)=g_{v}<\omega^{\mu}(x)>\gamma_{\mu}-g_{s}<\phi(x)>.
$$

In the first order of the gradient expansion, both RKE (27) and (40) lead to identical results in the frame of the mean field approximation:

$$
\begin{aligned}
& P^{\mu} \partial_{\mu}(x) G^{<}(x P)+\frac{1}{2} \partial^{\mu}(x) M(x)\left\{\hat{P}, \partial_{\mu}(P) G^{<}(x P)\right\} \quad+ \\
& +g_{v} P^{\mu} F_{\mu \nu}(x) \partial^{\nu}(P) G^{<}(x P)+\frac{1}{2}\left[\hat{P} \gamma^{\mu}, \partial_{\mu}(x) G^{<}(x P)\right] \quad+ \\
& +i M(x)\left[\hat{P}, G^{<}(x P)\right]-\frac{1}{2} g_{v} F_{\mu \nu}(x)\left[\hat{P} \gamma^{\mu}, \partial^{\nu}(P) G^{<}(x P)\right]=-S(x P),
\end{aligned}
$$

where $M(x)=m-g_{s}<\phi(x)>$ is the effective nucleon mass in the mean field approximation, $F^{\mu \nu}(x)=\partial^{\mu}<\omega^{\nu}>-\partial^{\nu}<\omega^{\mu}>$ and $P^{\mu}=p^{\mu}-g_{v}<\omega^{\mu}(x)>$ is the kinetic momentum.

RKE (46) converts in RKE of the Vlasov type when conditions of the collision effects are neglected $(S(x P)=0)$. The Vlasov RKE in this form was obtained and analyzed for the first time in Refs. [16]-17]. These equations are rather complicated because the spin and meson degrees of freedom as well as states with positive and negative energies are taken into account. On the basis of this RKE, a series of more simple, particular cases can be analyzed. The simplest situation corresponds to the case of a spin saturated system without antinucleon states, where all the spin-dependent effects in the RKE can 
be neglected (the spin effects were investigated separately in Ref. 16 ). Using the Clifford decomposition of the Wigner function, we get - in the quasi-classical limit - the wellknown RKE of the Vlasov type in the quantum hadrodynamics,

$$
P^{\mu} \partial_{\mu}(x) f^{S}(x P)+M \partial^{\mu}(x) M \partial_{\mu}(P) f^{S}(x P)+g_{v} P_{\mu} F^{\mu \nu} \partial_{\nu}(P) f^{S}(x P)=0,
$$

where $f^{S}(x P)$ corresponds to the scalar part of the Clifford decomposition of function $G^{<}(x P)$ (see Eq. (200). It is important that the basic RKE (46) leads to a correct RKE (47) of the Vlasov type for a spin saturated system without any additional assumption, like that of the rescaling procedure type (see the detailed discussion in [16]).

Technical details of derivation of Eqs.(46)-(47) are given in Refs. [16]-[17]. It is not difficult to obtain analogous RKE also without use of the gradient expansion. Such a RKE has a non-local character in the momentum space.

Calculation of collision integrals can be performed using two alternative schemes based either on formula (41) or (43). In the latter case, a standard perturbation theory was developed in Refs. [9, 10, 18, 19, 20]. Within the framework of the Walecka model (44), this approach allows us to derive collision integrals of the Bloch type (when the meson subsystem is considered on equal terms with the nucleon one) or of the Boltzmann type (if the role of mesons is confined only to the formation of the effective nucleon-nucleon interaction).

Let us return to the representation of the collision integral (41) based on the method of modified real-time Green's functions. Our subsequent transformations are based on the analogy with the nonrelativistic theory [24, 29]. From the definition (36), it follows that the Green's functions and mass operator satisfy the identities:

$$
\begin{aligned}
& G^{R}(x p)-G^{A}(x p)=G^{>}(x p)-G^{<}(x p), \\
& \Sigma^{R}(x p)-\Sigma^{A}(x p)=\Sigma^{>}(x p)-\Sigma^{<}(x p) .
\end{aligned}
$$

Using these relations, the collision integral (41) can be transformed into the form

$$
\begin{aligned}
S(x p) & =\frac{i}{2}\left\{\hat{p}\left[\Sigma^{>}(x p) G^{<}(x p)-\Sigma^{<}(x p) G^{>}(x p)\right]+\right. \\
& \left.+\left[G^{<}(x p) \Sigma^{>}(x p)-G^{>}(x p) \Sigma^{<}(x p)\right] \hat{p}\right\}+S^{o f f}(x p),
\end{aligned}
$$

where $S^{o f f}(x p)$ is the off-mass-shell part of the collision integral,

$$
\begin{aligned}
S^{\text {off }}(x p) & =\frac{i}{2}\left\{\hat{p} \Sigma^{<}(x p)\left[G^{R}(x p)+G^{A}(x p)\right]+\hat{p}\left[\Sigma^{R}(x p)+\Sigma^{A}(x p)\right] G^{<}(x P)-\right. \\
& \left.-G^{<}(x p)\left[\Sigma^{R}(x p)+\Sigma^{A}(x p)\right] \hat{p}-\left[G^{R}(x p)+G^{A}(x p)\right] \Sigma^{<}(x p) \hat{p}\right\} .
\end{aligned}
$$

Of course, the off-shell effects enter in the whole collision operator (49), but function (50) refers to them exclusively for the present aims. The separation into two parts, (49) and (50), is ascertained by means of spectral properties of the Green's functions and the mass operator. In our formalism, the corresponding spectral decompositions can be fulfilled in a covariant form (see Appendix B). This circumstance is a very desirable property of the relativistic kinetic theory which is intended for a description of macroscopic motions of a medium. Let us remark that the off-shell processes play the most important role in the relativistic nuclear physics of intermediate [49] and high [50] energies.

The generalized RKE (40) with the collision integral (49), (50) are the main results of this Section. It is worth remarking that a few other versions of generalized RKE have been previously obtained in the literature (see, e.g., [3] and [48]). We have in mind the cases of derivation of RKE for the Fermi subsystem based either on the Dirac type equations of 
motion for the field operator or on the equations of the Kadanoff-Baym type (33), (34). However, it is clear that this procedure contains some elements of an indetermination and arbitrariness on the stage of the construction of the motion equation for the Wigner function. In considered our case, the selection rule (39) has a reliable dynamical basis (see Sec. 2) which was tested in Refs. [17]-22]. Of course, the generalized RKE (40) can be obtained also by a direct way on the basis of Eq. (27), without using the rule (39), with a help of the Dyson equation and definitions of the type of Eqs. (17).

RKE (40), (49), and (50) have a more complicated matrix structure. This characteristic behavior arises by taking into account inner degrees of freedom which are intrinsic for the considered Fermi subsystem and, in particular, states with positive and negative energies. Indeed, the presence of $\gamma^{0}$ matrices before the time derivative in equations of the Kadanoff-Baym type (33) and (34) ensures the "direct" time movement for states of the spinor fields with positive energies and the "inverse" time direction for states with negative energies. But for the kinetic theory, it is characteristic the existence of a single time arrow. The rule (39) just ensures a covariant way of projection of Eqs. (33) and (34) on such a single time direction.

Finally, let us return once more to the role of the assumption $p^{2}>0$ (Assumption 1). A consecutive realization of this limitation leads to appreciable complication of the considered integrals. Similar restriction is absent in the traditional approach where an integration is carried out in the whole momentum space. In order to get traditional results in the frame of our formalism, it is necessary to implement the extension of obtained RKE into space-like region. There are three aspects of such a continuation:

1) into the integrals with respect to the momentum space,

2) into the time differential operations,

3) into the time integral operations.

The extension of the momentum integrals into the region, $p^{2}<0$, is trivial in the quasiparticle approximation (Sec. 4).

As for the derivation of the Vlasov RKE, the considered continuation does not lead to some changes of differential operations with respect to the time (e.g., to a change $\partial / \partial \tau$ by $i \partial / \partial \tau)$. Indeed, in the mean field approximation, the calculation of the force part of

the Vlasov RKE (the right-hand side in Eq.(27)) results in the cancellation of $\sqrt{p^{2}}$ once rule (25) has been used. In the other words, RKE conserves its physical content.

Finally, in the Markovian approximation, integral time operations do not lead to any modification of results at the continuation into the region $p^{2}<0$.

The possibility of continuation beyond the above-mentioned cases needs further researches.

\section{Quasiparticle approximation}

In this Section, we derive a covariant generalization of the quasiparticle approximation (QPA), widely used in the kinetic theory, which allows us to leave, by the simplest way, the framework of the usual perturbation theory. After that, we check possibilities of the suggested method on an example of derivation of collision integral of the BoltzmannUehling-Uhlenbeck (BUU) type for the standard Walecka model of relativistic nuclear matter consisting of nucleons, scalar and vector mesons with the interaction Hamiltonian (44). Finally, we discuss briefly a feasible generalization of the QPA in the model (44).

Let us introduce a non-equilibrium spectral function in the Wigner representation (see the first formula in Eq.(48))

$$
a(x p)=i\left[G^{R}(x p)-G^{A}(x p)\right]=i\left[G^{>}(x p)-G^{<}(x p)\right],
$$


This function satisfies the following sum rule

$$
(2 \pi)^{3} \int d E a\left(x ; p_{\perp}, E\right)=\gamma u,
$$

which is a simple consequence of the single-time anti-commutation relations for the field operators and of the rule (25). In Eq. (52), the integration is carried out over the longitudinal energy, $E=p n ; p_{\perp}^{\mu}=\Delta^{\mu \nu} p_{\nu}$ is the transverse momentum $\left(\Delta^{\mu \nu}\right.$ is the projection operator (9)).

$G^{>}(x p)$ and $G^{<}(x p)$, can be represented as:

$$
\begin{aligned}
G^{<}(x p) & =i a(x p) \mathcal{F}(x p), \\
G^{>}(x p) & =-i a(x p)[1-\mathcal{F}(x p)],
\end{aligned}
$$

where $\mathcal{F}(x p)$ is a unknown function.

To determine the non-equilibrium spectral function, we consider relation (51) and the corresponding motion equations for the retarded and advanced Green's functions. We write at once these equations in the minimal order of the gradient expansion, namely,

$$
\begin{array}{r}
{\left[\hat{p}+\frac{i}{2} \gamma \partial(x)-m-\Sigma_{M F}(x)+\frac{i}{2} \partial_{\mu}(x) \Sigma_{M F}(x) \partial^{\mu}(p)\right] G^{R / A}(x p)=} \\
=1+\Sigma^{R / A}(x p) G^{R / A}(x p) \\
G^{R / A}(x p)\left[\hat{p}-\frac{i}{2} \gamma \overleftarrow{\partial}(x)-m-\Sigma_{M F}(x)-\frac{i}{2} \overleftarrow{\partial}_{\mu}(p) \partial^{\mu}(x) \Sigma_{M F}(x)\right]= \\
=1+G^{R / A}(x p) \Sigma^{R / A}(x p) .
\end{array}
$$

Since the gradient expansion orders in the drift and collisions parts of RKE (40) are fixed, we can restrict ourselves to order zero of this decomposition in the estimation of correlation functions (53), so that [3],

$$
\left[\hat{p}-m-\Sigma_{M F}(x)-\Sigma^{R / A}(x p)\right] G^{R / A}(x p)=1 .
$$

In our toy model, $\Sigma_{M F}(x)$ is defined by Eq. (45) and hence,

$$
\left[\hat{P}-M(x)-\Sigma^{R / A}(x p)\right] G^{R / A}(x p)=1 .
$$

Let us assume now - as a working approximation - that the mass operator, $\Sigma(x p)$, has the same matrix structure as the mean field part (45) (in the Walecka model (44), this implies a possibility to neglect the tensor part of Clifford's decomposition of the mass operator),

$$
\Sigma(x p) \cong \Sigma_{s}(x p)+\gamma^{\mu} \Sigma_{\mu}(x p)
$$

(a more general form is given in Ref. 12]). In this expression as well as the following ones, the marks $R / A$ are omitted for simplicity's sake. Eq. (56) has then a matrix structure like the Dirac equation of noninteracting fields (quasiparticle structure)

$$
[\hat{\mathcal{P}}(x p)-\mathcal{M}(x p)] G^{R / A}(x p)=1,
$$

where

$$
\begin{gathered}
\mathcal{P}_{\mu}(x p)=P_{\mu}(x p)-\Sigma_{\mu}(x p), \\
\mathcal{M}(x p)=M(x p)-\Sigma_{s}(x p) .
\end{gathered}
$$

Therefore, Eq. (58) can be easily solved:

$$
G^{R / A}(x p)=\frac{\hat{\mathcal{P}}+\mathcal{M}}{\mathcal{P}^{2}-\mathcal{M}^{2} \pm i \epsilon E} .
$$


Then, from Eqs. (51) and (61), we get

$$
a(x \mathcal{P})=2 \pi(\hat{\mathcal{P}}+\mathcal{M}) \delta\left[\operatorname{Re}\left(\mathcal{P}^{2}-\mathcal{M}^{2}\right)\right]\{\theta(E)-\theta(-E)\} .
$$

This result is correct, provided that the quasiparticle excitations are weakly damped, i.e.,

$$
\left|\operatorname{Re}\left(\mathcal{P}^{2}-\mathcal{M}^{2}\right)\right|>>\left|\operatorname{Jm}\left(\mathcal{P}^{2}-\mathcal{M}^{2}\right)\right|
$$

It is easy to verify that the spectral function (62) satisfies the sum rule (52).

The substitution of the non-equilibrium spectral function (62) into Eqs. (53) leads to the following expressions for the correlation functions,

$$
\begin{aligned}
G^{<}(x \mathcal{P}) & =2 \pi i(\hat{\mathcal{P}}+\mathcal{M}) \delta\left(\mathcal{P}^{\prime 2}-\mathcal{M}^{\prime 2}\right) \mathcal{F}\left(x \mathcal{P}^{\prime}\right), \\
G^{>}(x \mathcal{P}) & =-2 \pi i(\hat{\mathcal{P}}+\mathcal{M}) \delta\left(\mathcal{P}^{\prime 2}-\mathcal{M}^{\prime 2}\right)\left[1-\mathcal{F}\left(x \mathcal{P}^{\prime}\right)\right],
\end{aligned}
$$

where $\mathcal{P}^{\prime}=\operatorname{Re} \mathcal{P}$ and $\mathcal{M}^{\prime}=\operatorname{Re} \mathcal{M}$.

As an illustration, we present a derivation of a collision integral of the BUU type in the Walecka model (44). This example is well known in the literature, therefore it is convenient for testing our method. We confined ourselves to the Born approximation only in the calculation of the self energy parts, $\Sigma^{>/<}$, in the collision integral (49). The corresponding diagrams are shown in Fig. 2.
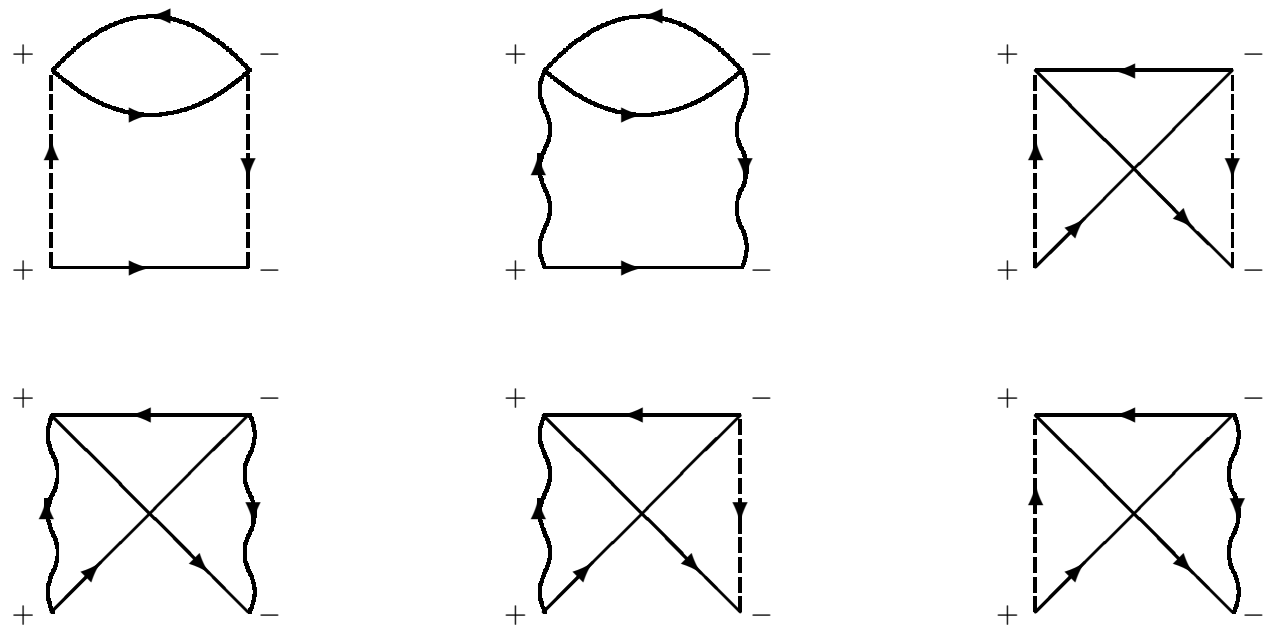

Fig. 2. Born diagrams for the self-energy part, $-i \Sigma^{<}=-i \Sigma^{-+}$. The solid, dashed and wavy lines denote the propagators of nucleons, scalar and vector mesons, respectively.

In a general case, this collision integral has a rather complicated form since we take into account simultaneously the spin and meson degrees of freedom and states with positive and negative energies. The simplest situation corresponds to a spin saturated nuclear matter without antinuclear component. In order to get the corresponding collision integral, we have to perform a transform from the spinor representation to the spin one for states with positive energies [26] and to take into account only the diagonal part of the Wigner function in the spin representation. As a result, for function $\mathcal{F}(x \mathcal{P})$ in Eq. (64), we obtain (for simplicity, we omit here and below all primes in functions $\mathcal{M}^{\prime}$ and $\mathcal{P}^{\prime}$ )

$$
\mathcal{F}_{\alpha \beta}(x \mathcal{P}) \Rightarrow \sum_{r, s=1,2} u_{\alpha}^{s} \bar{u}_{\beta}^{r} \Phi_{s r}(x \mathcal{P}) \Rightarrow \frac{(\hat{\mathcal{P}}+\mathcal{M})_{\alpha \beta}}{2 \mathcal{P}^{0}} \Phi(x \mathcal{P})
$$


where $\Phi_{s r}(x \mathcal{P})$ is the Wigner function of states with positive energy in the spin representation. For a spin-saturated nucleon subsystem we have $\Phi_{r s} \simeq \Phi \delta_{r s}$, where $\Phi(x \mathcal{P})$ is the scalar Wigner function. In Eq.(65), we used the completeness relation for the Dirac spinor [41]

$$
\sum_{r=1,2} u^{r} \bar{u}^{r}=\frac{\hat{\mathcal{P}}+\mathcal{M}}{2 \sqrt{\mathcal{M}^{2}+\overline{\mathcal{P}}^{2}}}
$$

Substitution of Eq. (65) into the first formula of Eqs. (64) leads to the relation

$$
G^{<(+)}(x \mathcal{P})=i \pi \frac{\hat{\mathcal{P}}+\mathcal{M}}{\mathcal{P}^{0}} \delta\left(\mathcal{P}^{0}-\sqrt{\mathcal{M}^{2}+\overline{\mathcal{P}}^{2}}\right) F(x \mathcal{P}),
$$

where a new auxiliary function is introduced:

$$
F(x \mathcal{P})=\frac{\mathcal{M}}{\mathcal{P}^{0}} \Phi(x \mathcal{P}) .
$$

This expression differs from the true Wigner function, $\Phi(x \mathcal{P})$, by the scaling factor, $\mathcal{M} / \mathcal{P}^{0}$ [42]. Introduction of such a rescaled Wigner function (67) is convenient in order to write the collision integral.

A tedious but straightforward evaluation of the on-mass-shell part of the collision integral (49) leads to the following result:

$$
\begin{aligned}
& S^{(+)}(x \mathcal{P})=(2 \pi)^{-5} \int \prod_{i=2}^{4} \frac{d^{3} \mathcal{P}_{i}}{\sqrt{\mathcal{M}^{2}+\overline{\mathcal{P}}_{i}^{2}}} W\left(\mathcal{P}, \mathcal{P}_{2}, \mathcal{P}_{3}, \mathcal{P}_{4}\right) \delta\left(\mathcal{P}-\mathcal{P}_{2}-\mathcal{P}_{3}-\mathcal{P}_{4}\right) \\
&\left\{F(x \mathcal{P}) F\left(x \mathcal{P}_{2}\right) \bar{F}\left(x \mathcal{P}_{3}\right) \bar{F}\left(x \mathcal{P}_{4}\right)-\bar{F}(x \mathcal{P}) \bar{F}\left(x \mathcal{P}_{2}\right) F\left(x \mathcal{P}_{3}\right) F\left(x \mathcal{P}_{4}\right)\right\}
\end{aligned}
$$

where $\bar{F}=1-F$ is the Pauli blocking factor. Here, the transition rates are defined as follows:

$$
W=W_{s s}+W_{v v}+W_{s v} .
$$

The partial transition rates are given by exchange of the corresponding pairs of the scalar or vector mesons:

$$
\begin{aligned}
W_{s s}\left(\mathcal{P} \mathcal{P}_{2} \mathcal{P}_{3} \mathcal{P}_{4}\right)= & 4 g_{s}^{4}\left\{4 D _ { s } ^ { 2 } ( \mathcal { P } - \mathcal { P } _ { 3 } ) \left[\mathcal{M}^{4}+\mathcal{M}^{2}\left(\mathcal{P} \mathcal{P}_{3}+\mathcal{P}_{2} \mathcal{P}_{4}\right)+\right.\right. \\
& \left.+\left(\mathcal{P} \mathcal{P}_{3}\right)\left(\mathcal{P}_{2} \mathcal{P}_{4}\right)\right]-D_{s}\left(\mathcal{P}-\mathcal{P}_{3}\right) D_{s}\left(\mathcal{P}-\mathcal{P}_{4}\right)\left[\mathcal{M}^{4}+\right. \\
& +\mathcal{M}^{2}\left(\mathcal{P} \mathcal{P}_{2}+\mathcal{P} \mathcal{P}_{3}+\mathcal{P} \mathcal{P}_{4}+\mathcal{P}_{2} \mathcal{P}_{3}+\mathcal{P}_{2} \mathcal{P}_{4}+\mathcal{P}_{3} \mathcal{P}_{4}\right)+ \\
& \left.\left.+\left(\mathcal{P}_{2} \mathcal{P}_{4}\right)\left(\mathcal{P} \mathcal{P}_{3}\right)+\left(\mathcal{P}_{2} \mathcal{P}_{3}\right)\left(\mathcal{P} \mathcal{P}_{4}\right)-\left(\mathcal{P} \mathcal{P}_{2}\right)\left(\mathcal{P}_{3} \mathcal{P}_{4}\right)\right]\right\} \\
W_{v v}\left(\mathcal{P} \mathcal{P}_{2} \mathcal{P}_{3} \mathcal{P}_{4}\right)= & 8 g_{v}^{4}\left\{4 D _ { v } ^ { 2 } ( \mathcal { P } - \mathcal { P } _ { 3 } ) \left[2 \mathcal{M}^{4}-\mathcal{M}^{2}\left(\mathcal{P}_{2} \mathcal{P}_{4}+\mathcal{P} \mathcal{P}_{3}\right)+\right.\right. \\
& \left.+\left(\mathcal{P} \mathcal{P}_{2}\right)\left(\mathcal{P}_{3} \mathcal{P}_{4}\right)+\left(\mathcal{P} \mathcal{P}_{4}\right)\left(\mathcal{P}_{2} \mathcal{P}_{3}\right)\right]- \\
& -D_{v}\left(\mathcal{P}-\mathcal{P}_{3}\right) D_{v}\left(\mathcal{P}-\mathcal{P}_{4}\right)\left[-2 \mathcal{M}^{4}+\mathcal{M}^{2}\left(\mathcal{P}_{2} \mathcal{P}_{3}+\mathcal{P} \mathcal{P}_{4}+\right.\right. \\
& \left.\left.\left.+\mathcal{P} \mathcal{P}_{3}+\mathcal{P} \mathcal{P}_{2}+\mathcal{P}_{3} \mathcal{P}_{4}+\mathcal{P}_{2} \mathcal{P}_{4}\right)-2\left(\mathcal{P} \mathcal{P}_{2}\right)\left(\mathcal{P}_{3} \mathcal{P}_{4}\right)\right]\right\} \\
W_{s v}\left(\mathcal{P} \mathcal{P}_{2} \mathcal{P}_{3} \mathcal{P}_{4}\right)= & 8 g_{s}^{2} g_{v}^{2}\left\{-4 D_{s}\left(\mathcal{P}-\mathcal{P}_{3}\right) D_{v}\left(\mathcal{P}-\mathcal{P}_{3}\right) \mathcal{M}^{2}\left[\mathcal{P} \mathcal{P}_{2}+\right.\right. \\
& \left.+\mathcal{P} \mathcal{P}_{4}+\mathcal{P}_{2} \mathcal{P}_{3}+\mathcal{P}_{3} \mathcal{P}_{4}\right]+D_{s}\left(\mathcal{P}-\mathcal{P}_{3}\right) D_{v}\left(\mathcal{P}-\mathcal{P}_{4}\right)\left[2 \mathcal{M}^{4}+\right. \\
& +\mathcal{M}^{2}\left(-\mathcal{P} \mathcal{P}_{2}+2 \mathcal{P P}_{3}-\mathcal{P} \mathcal{P}_{4}+2 \mathcal{P}_{2} \mathcal{P}_{4}-\mathcal{P}_{2} \mathcal{P}_{3}-\right. \\
& \left.\left.\left.-\mathcal{P}_{3} \mathcal{P}_{4}\right)+2\left(\mathcal{P} \mathcal{P}_{3}\right)\left(\mathcal{P}_{2} \mathcal{P}_{4}\right)\right]\right\}
\end{aligned}
$$

Finally, $D_{s, v}(\mathcal{P})$ are the Fourier transforms of the mesons Green's functions, $D_{s, v}(\mathcal{P})=$ $\left(\mathcal{P}^{2}-m_{s, v}^{2}\right)^{-1}$, and $m_{s}$ and $m_{v}$ are masses of the scalar and vector mesons, respectively. 
It is worth noticing that similar results have been recently obtained in Ref. [2] within the framework of non-covariant real-time Green's functions method. A comparison of our results with those of Ref. [2] shows an overall good agreement (up to a replacement of the fermionic Green function calculated in the mean-field approximation by that obtained in the more general approximation (64)). However, it needs to keep in mind that the standard Walecka model is too primitive for a proper description of real properties of nuclear matter at intermediate energies. Already at an equilibrium state, it leads to an equation of state stiffer than the expected one and, at moderately high density and temperature, the effective masses of nucleons become very small or even negative [33]. At present, two directions of the Walecka model improvement exist: either an increase of the number of its constituents [34] or a transition to a nonlinear generalization of the model [33, 35]. The first way leads to technical complications of the kinetic theory (by virtue of an essential increase of the number of constituents for real system), the second one remains still unexplored for a description of non-equilibrium states.

\section{Connection with the non-equilibrium statistical operator method}

As previously outlined, in the definition of Wigner's (11) and Green's functions (17), the statistical averaging was performed by means of the equilibrium density matrix, $\rho$, in the Heisenberg picture corresponding ordinarily to a system state at an infinite past. This fact limits the applicability of the theory to the case of weakly non-equilibrium states which can be described defining only slowly varying thermodynamic functions of the system such as temperature, chemical potential, etc. Now, let us discuss briefly a possibility of a generalization of the formalism suggested in Sec. 3 which is based on the change of the statistical averaging procedure, using the non-equilibrium statistical operator, $\rho(t)$. It is expected that such a generalization will allow us to describe strongly non-equilibrium states of a system [24, 29, 31, 32]. The non-equilibrium statistical operator method was adapted to fulfill requirements of the relativistic kinetic theory in our previous works [9,

10. 18, 19. Here, we discuss only a problem where this method is combined with the covariant formalism of real-time Green's functions method (Sec. 3).

Let us write the motion equation for the non-equilibrium statistical operator in a differential form 10

$$
d \rho(\tau) / d \tau=-\epsilon\left\{\rho(\tau)-\rho_{q}(\tau)\right\}
$$

and, in an integral form ( $\epsilon$ is an infinitely small value, $\epsilon>0$, and will approach to zero after the execution of the thermodynamic limiting transition),

$$
\rho(\tau)=\rho_{q}(\tau)+i \int_{-\infty}^{\tau} d \tau^{\prime} e^{\epsilon\left(\tau^{\prime}-\tau\right)}\left\{\left[\rho_{q}\left(\tau^{\prime}\right), H\left(\tau^{\prime}\right)\right]+i d \rho_{q}\left(\tau^{\prime}\right) / d \tau^{\prime}\right\} .
$$

Here, the time, $\tau$, is defined by Eq. (16) with a subsequent introduction of Assumptiones 1 and 2 and relation (12). An infinitely small source in the right-hand side of Eq. (71) is introduced to break the symmetry of this equation with respect to the time reflection (in the Wigner sense). The form of Eq. (71) allows us to select the retarded solution of the Liouville equation on the basis of an analogy with the formal scattering theory. This implies that after completing our calculations the thermodynamical limit should be taken and, then, the limit $\epsilon \rightarrow 0$ assumed.

The quasi-equilibrium statistical operator, $\rho_{q}(\tau)$, in Eq. (71) is the asymptotic form of the non-equilibrium statistical operator, $\rho(\tau)$, at the kinetic stage of evolution, when $\tau \rightarrow$ 
$-\infty$. An explicit form of operator $\rho_{q}(\tau)$ can be derived from the principle of the maximum of the information entropy under a supplementary condition of the given averaging value of $\left\langle P_{\alpha \beta}(x y)>_{q \tau}\right.$, where $<\ldots>_{q \tau}=\operatorname{Tr} \ldots \rho_{q}(\tau)$ and the operator, $P_{\alpha \beta}(x y)$, is defined by Eq. (2). For our task, it is convenient to associate the proper time, $\tau$, with the slow variable $x^{\mu}$ according to the expression (6).

As a result, we have [10]

$$
\rho_{q}(\tau)=\exp \{-S(\tau)\},
$$

where $S(\tau)$ is the entropy operator of the system at the kinetic stage,

$$
S(\tau)=\Phi(\tau)+\int d \sigma(x \mid u) \int d^{4} y P_{\alpha \beta}(x, y) F_{\alpha \beta}(x, y)
$$

and $\Phi(\tau)$ is the normalizing functional defined by condition $\operatorname{Tr} \rho_{q}(\tau)=1$. The Lagrange factor, $F_{\alpha \beta}(x, y)$, is determined by the self-consistency condition

$$
<P_{\alpha \beta}(x y)>_{\tau}=<P_{\alpha \beta}(x y)>_{q \tau}
$$

In the left-hand side of this equality, the averaging is performed by means of the nonequilibrium statistical operator $\rho(\tau)$, i.e., $\left\langle\ldots>_{\tau}=\operatorname{Tr} \ldots \rho(\tau)\right.$. Relation (75) shows a full equivalence of both statistical operators, $\rho(\tau)$ and $\rho_{q}(\tau)$, at the kinetic stage of evolution. Hence, both variants of the Wigner function,

$$
f_{\alpha \beta}(x, p)=(2 \pi)^{-4} \int d y e^{-i p y}<P_{\alpha \beta}(x, y)>_{\tau}=(2 \pi)^{-4} \int d y e^{-i p y}<P_{\alpha \beta}(x, y)>_{q \tau}
$$

can be used for a kinetic description of both quasi-equilibrium and non-equilibrium states.

The Green's functions, (16)-(19) and (36), also permit an analogous generalization for the case of strong non-equilibrium states.

$$
G_{(n o n-e q)}(x p)=-i(2 \pi)^{-4} \int d y e^{i p y}<T_{c}\left[\psi\left(x_{+}\right) \bar{\psi}\left(x_{-}\right)\right]>_{\tau} .
$$

The form of the Kadanoff-Baym Eqs. (33), (34) remains also the same, taking into account that the $\epsilon$-terms generated by the right-hand of Eq. (72) vanish after a realization of the thermodynamical limit. However, a restriction to the lowest terms of the gradient decomposition is incompatible with a description of strong non-equilibrium states. Therefore, the corresponding results of Sec. 4 require a further generalization. This field of relativistic kinetic theory is still scarcely investigated.

Let us remark that Eq. (72) can be rewritten in a form convenient for the construction of an iteration scheme under the energy of particle-particle interactions. In full analogy with the non-relativistic case [24], we obtain [9, 10]

$$
\begin{gathered}
\rho(\tau)=\rho_{q}(\tau)+i \int_{-\infty}^{\tau} d \tau^{\prime} e^{\epsilon\left(\tau^{\prime}-\tau\right)}\left\{\left[\rho_{q}\left(\tau^{\prime}\right), H_{i n}\left(\tau^{\prime}\right)\right]+\right. \\
\left.+\int d \sigma\left(x^{\prime} \mid u\right) \int d^{4} y^{\prime}<\left[P_{\alpha \beta}\left(x^{\prime}, y^{\prime}\right), H_{i n}\left(\tau^{\prime}\right)\right]>_{q \tau^{\prime}} \frac{\delta \rho_{q}\left(\tau^{\prime}\right)}{\delta<P_{\alpha \beta}\left(x^{\prime}, y^{\prime}\right)>_{\tau^{\prime}}}\right\} .
\end{gathered}
$$

As shows our experience, the second addend in the right-hand side of Eq. (78) removes usually all disconnected diagrams of a given order from the set of diagrams which are supplied by the first addend.

Some problems of this approach arise on the level of the non-equilibrium thermodynamical Wick's theorem. However, there is a set of methods which permits to overcome these difficulties [24, 45]. c 


\section{Conclusions}

In the present work, we have demonstrated an interesting possibility for a covariant generalization of the real-time Green's functions method which opens new ways after a transition to the Wigner representation. In fact, this generalization is based on the admissibility of introduction in every point of the Minkowski space a unit time-like vector constructed with the momentum vector of the corresponding point of the phase space. Therefore, for a definition of the preferred time-like direction in the Minkowski space, we used a possibility contained in the Wigner function itself. The relevant Assumptiones (Sec. 2) allow us to write, starting from the Heisenberg representation, a motion equation in a covariant form for the one-particle Wigner function (Sec. 2) and, after that, to obtain a generalized RKE in the frame of the Kadanoff-Baym technique (Sec. 3). Such a way is found especially effective for a kinetic description of subsystems with inner degrees of freedom and allows us to eliminate some ambiguities of relativistic kinetic theory based on the Kadanoff-Baym equations.

As a test of the suggested approach, the Fermi sector of the well known Walecka model of the relativistic nuclear matter was chosen (Sec. 3 and 4). For this model, RKE of the Vlasov type (Sec. 3) and of the BUU type (Sec. 4) were obtained. In the latter case, the collision integral was derived only for a spin saturated system without antinucleon states. The calculations were performed within the framework of the quasiparticle approximation (QPA). We discuss also a possibility of an extension of this approximation. An agreement with a set of well-known simple results in the literature denotes a noncontradictory character of the proposed generalizations. It is safe to assume that methods suggested here provide also a correct description of spin degrees of freedom, states with negative energies (e.g., for the annihilation channel in the theory of non-equilibrium electron-positron plasma), and so on.

Finally, the covariant modification of the real-time Green's functions method suggested in Sec. 3 was generalized to a kinetic description of strongly non-equilibrium states (Sec. 5). For this aim, we suggest to use the non-equilibrium statistical operator method.

The non-equilibrium relativistic nuclear matter represents a good example of very complicated object for theoretical and experimental investigations. Of course, the simplest toy model and our present results discussed in Sec. 4 are only a rough approximation of the reality. At present, a lot of actual problems still remain in the relativistic kinetic theory, e.g., going beyond the quasiparticle approximation, taking into account nonlocal (and, in particular, non-Markovian) effects in kinetic processes, cluster decomposition, etc. (see, e.g., 11, 27, 28, 36, 37, 38, 43, 44, 46]). An important feature of the examined approach is a lack of some dynamical constraints into a corresponding quantum field theory (these constraints are introduced in order to eliminate "unnecessary", non-physical degrees of freedom). The dynamical introduction of these constraints into the Kadanoff-Baym formalism is a rather non-trivial problem. A necessity to consider such constraints creates serious problems for the kinetic theory (e.g., in order to describe the $\Delta$-isobar subsystem of nuclear matter). From our point of view, the approach suggested in the present work can serve as a reliable basis for such kind of further researches in the relativistic kinetic theory.

\section{Acknowledgments}

We wish to thank V. G. Morozov for stimulating discussions and S. Mrówczyński for addressing our attention to some questions discussed here. 
This work was partly supported by the State Committee of Russian Federation for Higher Education under grant N 97-0-6.1-4 and was completed under the auspices of the U.S. Department of Energy by the Los Alamos National Laboratory under contract no. W-7405-ENG-36.

One from authors (S.A.S.) thanks the Soros Education Program for support.

\section{Appendices}

\section{A Proof of Eq. (25)}

Let us derive a proof of the Eq. (25) using a boost transformation.

$$
\int d^{3} z^{\prime} S\left(\mathbf{z}-\mathbf{z}^{\prime}, 0\right) f\left(z^{\prime}\right)=i \gamma^{0} f(z),
$$

for an arbitrary function of the field operator $f(z)$. The integration here is fulfilled over hyperplane of the constant time, $z^{0}=z^{\prime 0}$. Let us perform a transition to an arbitrary frame of reference using a transformation from the homogeneous Lorentz group

$$
x^{\mu}=\Lambda_{\nu}^{\mu}(u) z^{\nu}, \quad x^{\prime \mu}=\Lambda_{\nu}^{\mu}(u) z^{\prime \nu} .
$$

The velocity vector, $u^{\mu}$, defines simultaneously the orientation of the hyperplane, $\sigma(u)$. The transformation of the commutator function, $S\left(\mathbf{z}-\mathbf{z}^{\prime}, 0\right)$, in Eq. (A1) is obtained using the corresponding unitary operator, $U(\Lambda)$ [39],

$$
S\left(\mathbf{z}-\mathbf{z}^{\prime}, 0\right)=S\left[\Lambda\left(x-x^{\prime}\right)\right]=U(\Lambda) S\left(x-x^{\prime}\right) U^{-1}(\Lambda),
$$

where $x, x^{\prime} \in \sigma(u)$. Similarly, we have:

$$
f(z)=U(\Lambda) f(x) U^{-1}(\Lambda) .
$$

Let us take into account also the relation

$$
U^{-1}(\Lambda) \gamma^{\mu} U(\Lambda)=\Lambda_{\nu}^{\mu}(u) \gamma^{\nu}
$$

This equality is a consequence of the invariance under the Lorentz transformation (A2) of the scalar product of $\psi(x)$ and $\phi(x)$ [39],

$$
(\psi, \phi)_{\sigma}=\int d \sigma^{\mu}(x \mid u) \bar{\psi}(x) \gamma_{\mu} \phi(x) .
$$

Finally, it is necessary to consider the equalities $\Lambda_{0}^{0}(u)=u^{0}$ and $\Lambda_{k}^{0}(u)=u^{k}(k=1,2,3)$. Formulas (A2)-(A3) allow us to realize the transformation from Eq. (A1) to Eq. (25). Another proof of Eq. (25) can be found in Ref. [10].

\section{B Spectral properties of the modified Green's functions}

The spectral representations of the covariant contour Green's functions introduced in Sec. 3 can be also considered in a covariant form (we follow the work [3] were a similar approach was fulfilled in the framework of a non-covariant formalism). The main feature of these representations is the selection of the time arguments in the form (15). As an example, let 
us consider the spectral decomposition of the retarded and advanced Green's functions. According to the definitions, (16) and (36), we have (see the last remark in Sec. 3.2)

$$
G^{R / A}(x p)= \pm(2 \pi)^{-4} \int d y e^{i p y}\left\{G^{>}\left(x_{+}, x_{-}\right)-G^{<}\left(x_{+}, x_{-}\right)\right\} \theta\left[ \pm y_{\mu} u^{\mu}\right]
$$

where $u^{\mu}$ is the unit vector (12). From here, it follows

$$
G^{R / A}(x p)= \pm \frac{1}{2 \pi i} \int d^{4} p^{\prime} \int_{-\infty}^{\infty} d \eta \delta^{(4)}\left(p^{\mu}-p^{\prime \mu}+u^{\mu} \eta\right) \frac{G^{>}\left(x p^{\prime}\right)-G^{<}\left(x p^{\prime}\right)}{ \pm \eta-i \varepsilon}
$$

or

$$
G^{R / A}(x p)= \pm \frac{1}{2}\left[G^{>}(x p)-G^{<}(x p)\right]+\widetilde{G}(x p),
$$

where

$$
\widetilde{G}(x p)=\frac{1}{2 \pi i} \int d^{4} p^{\prime} \int_{-\infty}^{\infty} d \eta \delta^{(4)}\left(p^{\mu}-p^{\prime \mu}+u^{\mu} \eta\right)\left[G^{>}\left(x p^{\prime}\right)-G^{<}\left(x p^{\prime}\right)\right] \mathcal{P}\left(\frac{1}{\eta}\right) .
$$

Let us transform the last relation using the decomposition of an arbitrary vector, $a^{\mu}$, on the time-line direction, $u_{\mu}$ (12), and orthogonal to it space-like component,

$$
a^{\mu}=u^{\mu}(a u)+\Delta_{\nu}^{\mu} a^{\nu} \equiv a_{\|}^{\mu}+a_{\perp}^{\mu},
$$

where the projection operator, $\Delta^{\mu \nu}$, is defined by Eq. (9). Then, we can write the following representation

$$
\delta^{(4)}\left(p^{\mu}-p^{\prime \mu}+u^{\mu} \eta\right)=\delta\left[n_{\mu}\left(p^{\mu}-p^{\prime \mu}+u^{\mu} \eta\right)\right] \delta_{\sigma}\left(p_{\perp}^{\mu}-p_{\perp}^{\prime \mu}\right),
$$

where function $\delta_{\sigma}\left(p_{\perp}^{\mu}\right)$ is defined on the space-like hyperplane $\sigma(u)$. Using Eq. (B3), we can rewrite Eq. (B2) in the final form:

$$
\widetilde{G}(x p)=\frac{1}{2 \pi i} \int_{-\infty}^{\infty} d \omega \mathcal{P}\left(\frac{1}{\omega-\sqrt{p^{2}}}\right)\left\{G^{>}\left(x ; p_{\perp}, \omega\right)-G^{<}\left(x ; p_{\perp}, \omega\right)\right\} .
$$

The denominator, $\left(\omega-\sqrt{p^{2}}\right)$, in Eq. (B4) shows clearly that function $\widetilde{G}(x p)$ is just the off-mass-shell part of the Green's functions, $G^{R / A}(x p)$. As far as we have

$$
G^{R}(x p)+G^{A}(x p)=2 \widetilde{G}(x p),
$$

and an analogous relation for the mass operator, $\Sigma^{R / A}$, the last term in Eq. (49) represents indeed the off-mass-shell part of the collision integral (48). 


\section{References}

[1] W. Botermans and R. Malfliet, Phys. Rep. 198 (1990) 115.

[2] M. Schönhofen, M. Cubero, B. L. Friman, W. Nörenberg, and G. Wolf, Nucl. Phys. A572 (1994) 112.

[3] S. Mrówczyński and U. Heinz, Ann. Phys. (N.Y.) 229 (1994) 1.

[4] J. E. Davis and R. J. Perry, Phys. Rev. C 43 (1991) 1893.

[5] R. Bertoncini, Int. J. Mod. Phys. B 6 (1992) 3441.

[6] D. N. Voskresensky, D. Blaschke, G. Röpke, and H. Schulz, Int. J. Mod. Phys. E 4 (1995) 1.

[7] W. Cassing and U. Mosel, Prog. Part. Nucl. Phys. 25 (1990) 235.

[8] Sh.-J. Wang, B.-A. Li, W. Bauer, and J. Randrup, Ann. Phys. (N.Y.) 209 (1991) 251.

[9] S. V. Erokhin, A. V. Prozorkevich, S. A. Smolyansky, and V. D.. Toneev, J. Theor. Math. Phys. 95 (1993) 74.

[10] V. D. Toneev, A. V. Prozorkevich, and S. A. Smolyansky, Heavy Ion Phys. 3 (1996) 37.

[11] E. Calzetta and L. Hu, Phys. Rev. D 37 (1988) 2778.

[12] P. A. Henning, Phys. Rep. 253 (1995) 1.

[13] J. Rau and B. Müller, Phys. Rep. 272 (1996) 1.

[14] P. J. Siemens, M. Soyer, G. D. White, L. J. Latto, and K. T. R. Davies, Phys. Rev. C 40 (1989) 2641.

[15] A. Dellafiore and F. Matera, Phys. Rev. C 44 (1991) 2456.

[16] S. G. Mashnik, A. V. Prozorkevich, S. A. Smolyansky, and G. Maino, Nuovo Cim. A 109 (1996) 1699;

G. Maino, S. G. Mashnik, A. V. Prozorkevich, and S. A. Smolyansky Izv. Akad. Nauk, Ser. Fiz. 62 (1998) 1004.

[17] S. A. Smolyansky, A. V. Prozorkevich, S. Schmidt, D. Blaschke, G. Röpke, and V. D. Toneev, Int. J. Mod. Phys. E 7 (1998) 515.

[18] A. V. Prozorkevich and S. A. Smolyansky, in Selected Topics of Nuclear Physics, p. 127, JINR, Dubna, Russia, 1995.

[19] A. V. Prozorkevich, S. A. Smolyansky, and V. D. Toneev, JINR preprint P 7-95-362, Dubna, Russia (1995).

[20] A. V. Prozorkevich, S. A. Smolyansky, and V. D. Toneev, Yad. Fiz. 59 (1996) 804 [Phys. At. Nucl. 59 (1996) 766].

[21] G. Maino, S. G. Mashnik, S. A. Smolyansky, A. V. Tarakanov, and D. Tocci, Izv. Akad. Nauk, Ser. Fiz. 60 (1996) 58 [Bull. Russian Academy of Sci. 60 (1996) 731].

[22] S. A. Smolyansky, A. V. Prozorkevich, S. G. Mashnik, and G. Maino, in Proc. Int. Conf. on Nucl. Data for Science and Technology, Trieste, Italy, May 19-24, 1997, SIF Proceedings, vol.59, part I, p. 325, Bologna, 1997.

[23] P. T. Matthews, Phys. Rev. 75 (1949) 1270; N. M. Kroll, ibid, 1321; V. G. Kadyshevsky, J. Exp. Theor. Phys. (U.S.S.R.) 46 (1964) 654 [Sov. Phys. JETP 19 (1964) 443]; N. M. Atakishev, R. M. Mir-Kassimov, and Sh. M. Nagiev, Theor. Mat. Fiz. 32 (1977) 34; E. A. Dey, V. N. Kapshay, and N. B. Skachkov, ibid, 101 (1994) 69. 
[24] D. N. Zubarev, V. G. Morozov, and G. Röpke, Statistical Mechanics of Nonequilibrium Processes, Vol. 2, Akademie Verlag GmbH, Berlin, 1997.

[25] P. Danielewicz, Ann. Phys. (N.Y.) 152 (1984) 239.

[26] S. R. de Groot, W. A. van Leeuwen, and Ch. G. van Weert, Relativistic Kinetic Theory, North-Holland, Amsterdam, 1980.

[27] S. Chattopadhyay and D. Pal, J. Phys. G: Nucl. Part. Phys. 20 (1994) 357.

[28] H. S. Köhler, Phys. Rev. C 51 (1995) 3232.

[29] L. P. Kadanoff and G. Baym, Quantum Statistical Mechanics, Benjamin, New York, 1962.

[30] B. D. Serot and J. D. Walecka, Adv. Nucl. Phys. 16 (1986) 1.

[31] J. A. McLennan, Non-Equilibrium Statistical Mechanics, New Jersey, Prentice Hall, 1989.

[32] D. N. Zubarev, V. G. Morozov, and G. Röpke, Statistical Mechanics of Nonequilibrium Processes, Vol. 1, Akademie Verlag GmbH, Berlin, 1996.

[33] J. Zimanyi and S. A. Moszkowski, Phys. Rev. C 42 (1990) 1416.

[34] P. Bernardos, V. N. Fomenko, N. V. Giai, M. L. Quelle, S. Marcos, R. Niembro, and L. N. Savushkin, Phys. Rev. C 48 (1993) 2665; V. N. Fomenko, S. Marcos, and L. N. Savushkin, J. Phys. G: Nucl. Part. Phys. 19 (1993) 545.

[35] A. Delfino, C. T. Coelcho, and M. Malheiro, Phys. Rev.. C 51 (1995) 2188.

[36] V. M. Kolomiets, A. G. Magner, and V. A. Plujko, Yad. Fiz. 55 (1992) 2061 [Sov. J. Nucl. Phys. 55 (1992) 1143].

[37] G. Röpke, H. Schulz, K. K. Gudima, and V.D.Toneev, Fiz. Elem. Chastits At. Yadra 21 (1990) 364 [Sov. J. Part. Nucl. 21 (1990) 153].

[38] Th. Bornath, D. Kremp, W. D. Kraeft, and M.Schlanges, Phys. Rev. E 54 (1996) 3274 .

[39] S. S. Schweber, An Introduction to Relativistic Quantum Field Theory, Row, Peterson and Co, New York, 1961.

[40] N. P. Landsman and C. G. van Weert, Phys.Rep. 145 (1987) 141.

[41] J. D. Bjorken and S. D. Drell, Relativistic Quantum Fields, Mc Graw-Hill, San Francisco, 1964.

[42] H. -Th. Elze, M. Gyulassy, D. Vasak, U. Heinz, H. Stöcker, and W. Greiner, Mod. Phys. Lett. A2 (1987) 451.

[43] P. Král, J. Stat. Phys. 86 (1997) 1337.

[44] H. S. Köhler and R. Malfliet, Phys. Rev. C 48 (1993) 1034.

[45] R. Fauser and H. H. Wolter, Nucl. Phys. A 600 (1996) 491.

[46] D. Kremp, M. Bonitz, W. D. Kraeft, and M. Schlanges, Ann. Phys. (N.Y.) 258 (1997) 320 .

[47] D. N. Zubarev, Non-equlibrium Statistical Thermodynamics, Plenum Press, New York, 1974.

[48] S. Mrówczyński and U. Heinz, reprint TPR-92-3\%.

[49] Yu. B. Ivanov, J. Knoll and D. N. Voskresensky, report hep-ph/9807351;

P. Bozek, Phys. Rev. C 56 (1997) 1452. 
[50] B. Müller, report nucl-th/9807042;

K. Geiger and B. Müller, Heavy Ion Physics 7 (1998) 207;

K. Geiger, Phys. Rev. D 54 (1996) 949; 56 (1997) 2665.

D. Boyanovsky, H. de Vega, R. Holman, S.Kumar, R. Pisarsky, report hep$\mathrm{ph} / 9802370$. 